\title{
Mixed collective states of many spins
}

\author{
Janus Wesenberg and Klaus Mølmer \\ QUANTOP, Danish Quantum Optics Center, Institute of Physics and Astronomy, \\ University of Aarhus, DK-8000 Århus C, Denmark
}

(Dated: October 29, 2018)

\begin{abstract}
Mixed states of samples of spin $s$ particles which are symmetric under permutations of the particles are described in terms of their total collective spin quantum numbers. We use this description to analyze the influence on spin squeezing due to imperfect initial state preparation.
\end{abstract}

PACS numbers: 03.67.-a, 42.50.-p

\section{INTRODUCTION}

Atomic samples for which external perturbations and mutual interactions between the atoms are completely symmetric under permutations of the atoms are conveniently described in terms of collective variables. In particular, if the atoms have two internal states relevant for the dynamical processes, each atom can be represented as a spin $\frac{1}{2}$ particle, and the full Hamiltonian of the system can be expressed in terms of the collective spin operator $\boldsymbol{J}=\sum_{i} \boldsymbol{S}_{i}$. A state vector of the system which reflects the permutation symmetry of $n$ two-level particles can be expanded on the set of symmetric Dicke states $\left|\begin{array}{c}j_{0} \\ m\end{array}\right\rangle$, where $j_{0}=n / 2$, and the quantum number $m$ ranges from $-j_{0}$, where all the atoms populate the states associated with a spin down single particle state, to $j_{0}$ where all are in the spin up state. Intermediate values represent states containing all possible permutations of the atoms populating either of the two spin $\frac{1}{2}$ components, but with a definite total number, $j_{0} \pm m$, of atoms in the spin up $(+)$ and down states $(-)$.

Collective behavior of atomic ensembles is at the root of superradiance, and the Dicke states were introduced to account for atom-field interactions already many years ago. The past decade has provided numerous discussions and analyses of collective atomic dynamics in connection with atomic clocks and quantum information [1, 2], and recently a number of promising schemes has been presented for tailoring of the collective atomic spin noise, relying on atom-light interaction [3, 4, collisional interactions in atomic samples [5, 6, 7], and on quantum nondemolition measurements $[8,9]$.

Restricting calculations to the space spanned by the symmetric Dicke states implies a formidable simplification of the full $2^{n}$ dimensional problem to an $n+1$ dimensional one, and use of algebraic properties of the angular momentum operators further facilitate calculations of properties of the physical system. A large number of theoretical analyses therefore presents calculations restricted to this subspace. But, even if the assumption holds that the systems is invariant under exchange of atoms, not only the symmetric Dicke states will be populated: Imagine two two-level atoms, both described by the mixed state density matrix

$$
\rho_{1}=p|\uparrow\rangle\langle\uparrow|+(1-p)| \downarrow\rangle\langle\downarrow|
$$

The combined state $\rho_{2}=\rho_{1} \otimes \rho_{1}$ of both atoms is also a mixed state. It is manifestly symmetric under exchange of the atoms, but if $p \neq 0$ or 1 it is a mixture of all $2^{2}=4$ product states spanning the full Hilbert space of the pair of particles, and by a basis change we see that both the spin singlet and the three spin triplet eigenstates of the collective spin operator are populated. Unlike the spin singlet wave function, a density matrix component proportional to a projector on the spin singlet state is, indeed, invariant under exchange of the particles.

Symmetric mixed states incoherently populate state vectors of different permutation symmetries. It is the purpose of the present paper to characterize this population of states of lower symmetry and to investigate the effect, e.g., on the achievements of spin squeezing and multi-particle entanglement of samples of atoms. For experiments, the situation with values of $p$ close to zero or unity are particularly interesting as they represent the degree of polarization obtained, e.g., by optical pumping.

In section II we derive some expectation values for the collective spin operators. In section III, we derive the probability distribution for states of different $j$ and $m$, $j \leq j_{0}$, for a collection of spin $\frac{1}{2}$ particles in the state (11). In section IV, we analyze the achievements of spin squeezing on such a collection of particles. In section $\mathrm{V}$ we discuss the case of $s>\frac{1}{2}$, i.e., atoms with more than two states.

\section{EXPECTATION VALUES OF THE COLLECTIVE SPIN OPERATORS}

Before determining the full density matrix of a system of uncorrelated atoms, it is instructive to see how much information can be derived from the fact that the atoms are uncorrelated.

For a system of $n$ uncorrelated atoms, we may determine the variances of the collective spin operators through

$$
\Delta J_{\alpha}^{2}=n \Delta S_{\alpha}^{2} \quad \alpha=x, y, z .
$$

In the case where the individual atoms are two-level atoms distributed according to (11), we obviously have $\left\langle\boldsymbol{S}^{2}\right\rangle=\frac{3}{4}$ and $\left\langle S_{z}^{2}\right\rangle=\frac{1}{4} p+\frac{1}{4}(1-p)=\frac{1}{4}$, assuming $\hbar=1$. Since the distribution is invariant under rotations 
around the $z$-axis, the $x$ - and $y$-variances must be equal and we find

$$
\Delta J_{x}^{2}=\Delta J_{y}^{2}=\frac{n}{2}\left\langle\boldsymbol{S}^{2}-S_{z}^{2}\right\rangle=\frac{1}{2} j_{0} .
$$

Also, since the expectation value of $S_{z}$ is $p-\frac{1}{2}$, the variance of $J_{z}$ is

$$
\Delta J_{z}^{2}=n\left(\left\langle S_{z}^{2}\right\rangle-\left\langle S_{z}\right\rangle^{2}\right)=p(1-p) 2 j_{0} .
$$

To find $\left\langle\boldsymbol{J}^{2}\right\rangle$ we may again utilize the fact that the atoms are independent so that $\left\langle\boldsymbol{J}^{2}\right\rangle=\sum_{i \neq j}\left\langle\boldsymbol{S}_{i}\right\rangle \cdot\left\langle\boldsymbol{S}_{j}\right\rangle+$ $\sum_{i}\left\langle\boldsymbol{S}_{i}^{2}\right\rangle$, which in this case simplifies to

$$
\left\langle\boldsymbol{J}^{2}\right\rangle=(2 p-1)^{2} j_{0}\left(j_{0}+1\right)+6 p(1-p) j_{0} .
$$

In the completely polarized case, $p=1$, the total spin equals $j_{0}=n / 2$ and $\left\langle\boldsymbol{J}^{2}\right\rangle=j_{0}\left(j_{0}+1\right)$. For mixed ensembles $\left\langle\boldsymbol{J}^{2}\right\rangle$ is reduced, reflecting a distribution over states with different $j$, and in the completely unpolarized case, $p=\frac{1}{2}$, each atom contributes $\frac{3}{4}$ to $\left\langle\boldsymbol{J}^{2}\right\rangle=n \frac{3}{4}$. In the following section we shall determine the population of states with different $j$ for any value of $p$.

\section{THE DENSITY MATRIX FOR A TENSOR PRODUCT OF MIXED STATES}

$n$ spin $\frac{1}{2}$ particles span a $2^{n}$ dimensional Hilbert space, $\mathcal{H}$, with basis vectors $\left|m_{1}, \ldots, m_{n}\right\rangle, m_{i}= \pm \frac{1}{2}$. An alternative representation is by means of eigenstates, $\left|{ }_{m}^{j} ; \lambda\right\rangle$, of the collective spin operators $\boldsymbol{J}^{2}$ (eigenvalue $j(j+1)$ ) and $J_{z}$ (eigenvalue $m$ ), where $\lambda$ enumerates the degenerate states within the eigenspaces, $\mathcal{H}_{j, m}$, of the $\boldsymbol{J}^{2}$ and $J_{z}$ operators.

\section{A. Collective spin states for $n$ spin $\frac{1}{2}$ particles}

A complete description of the full set of basis states is given in Ref. [10], but for completeness we shall derive our results by a formally simpler analysis.

The $\left|{ }_{m}^{j} ; \lambda\right\rangle$ states must span the full $2^{n}$ dimensional Hilbert space, and we start out by identifying the number of states with a given value of the quantum number $m$. Since $m=\sum_{i} m_{i}$, the total number of atoms in the spin up state $|\uparrow\rangle$ is $j_{0}+m$, and these states can be selected in a number of ways given by the combinatorial factor

$$
N_{m}=\left(\begin{array}{c}
n \\
j_{0}+m
\end{array}\right)=\frac{n !}{\left(j_{0}+m\right) !\left(j_{0}-m\right) !} .
$$

Due to the binomial formula $(1+1)^{n}=\sum_{k}\left(\begin{array}{l}n \\ k\end{array}\right) 1^{k} 1^{(n-k)}$ we verify that $\sum_{m} N_{m}=2^{n}$.

States with given $m$ values should now be grouped according to the values of $j$. Clearly $j \geq m$, hence we may begin with $m=j_{0}$ for which only a single state exists, and for which $j$ must equal $j_{0}$. The combinatorial factor $N_{m=j_{0}-1}$ equals $n$ (a single spin down particle can be chosen in $n$ different ways), and since only one of the states with $m=j_{0}-1$ can be obtained by the collective lowering operator $J_{-}$acting on the state $\left|j_{0} j_{0}\right\rangle$, the remaining $n-1$ states must have $j=j_{0}-1$. Applying the lowering operator $J_{-}$on all the identified $m=j_{0}-1$ states produces one state with $j=j_{0}$ and $n-1$ states with $j=j_{0}-1$. The remaining $N_{m=j_{0}-2}-n$ must therefore have $j=j_{0}-2$. Continuing this argument provides the total number of states with $j \geq m$ for any $m \geq 0$, and by symmetry we obtain the same number of $j$-states for negative $m$. In conclusion, the number of states with given values of $j$ and $m$ is independent of $m$ and equal to $D_{j}^{(1 / 2)}=N_{m=j}-N_{m=j+1}$ :

$$
\operatorname{dim} \mathcal{H}_{j, m}=D_{j}^{(1 / 2)}=\frac{2 j+1}{j_{0}+j+1}\left(\begin{array}{c}
2 j_{0} \\
j_{0}+j
\end{array}\right) .
$$

\section{B. The density matrix for a tensor product of mixed states}

Consider now the n-particle density operator

$$
\rho_{n}=\rho_{1}^{\otimes n}
$$

i.e., the tensor product of $n$ identical two-state density matrices. Since any two-state density matrix may be diagonalized through rotations, we will only consider the diagonal form of $\rho_{1}$ as given by (11).

As we intend to demonstrate the usefulness of the collective spin operator, we will only be interested in the reduced density matrix, $\rho_{n}^{\text {red }}$, obtained by taking the partial trace over the remaining degrees of freedom:

$$
\left\langle\begin{array}{c}
j^{\prime} \\
m^{\prime}
\end{array}\left|\rho_{n}^{\text {red }}\right| \begin{array}{c}
j \\
m
\end{array}\right\rangle \equiv \sum_{\lambda}\left\langle\begin{array}{c}
j^{\prime} \\
m^{\prime}
\end{array} ; \lambda\left|\rho_{n}\right| \underset{m}{j} ; \lambda\right\rangle .
$$

To determine the $p$-dependence of the matrix elements of $\rho_{n}^{\text {red }}$, we note that the vector $\left|{ }_{m}^{j} ; \lambda\right\rangle$ may be expanded on the product basis, $\left\{\left|m_{1}, m_{2}, \ldots, m_{n}\right\rangle\right\}$, so that (9) only involves matrix elements of the form

$$
\left\langle m_{1}^{\prime}, m_{2}^{\prime}, \ldots, m_{n}^{\prime}\left|\rho_{1}^{\otimes n}\right| m_{1}, m_{2}, \ldots, m_{n}\right\rangle,
$$

where $\sum m_{i}^{\prime}=m^{\prime}$ and $\sum m_{i}=m$. Since $\rho_{1}$ is diagonal, only matrix elements with $m_{i}^{\prime}=m_{i}$ are non-vanishing, and consequently $\rho_{n}^{\text {red }}$ is diagonal in $m$. Writing the diagonal elements of $\rho_{1}$ as $p^{1 / 2+m_{s}}(1-p)^{1 / 2-m_{s}}$, it is evident that the value of any non-vanishing matrix element of the form 10 is $p^{j_{0}+m}(1-p)^{j_{0}-m}$. Since the expansions coefficients used to write $\rho_{n}^{\text {red }}$ in terms of these matrix elements are independent of $p$, we conclude that this is the full $p$-dependence of the matrix elements of $\rho_{n}^{\text {red }}$.

We now consider the case where $p=\frac{1}{2}$, so that the single particle density matrix, $\rho_{1}$, is proportional to the identity. This implies that the full density matrix (8) is also proportional to the identity, or to be more specific 
$\rho_{n}=1 / \operatorname{dim} \mathcal{H}$ in any basis. Consequently, since the entries of $\rho_{n}^{\text {red }}$ are obtained by performing a partial trace as defined in (9),$\rho_{n}^{\text {red }}$ is diagonal, and the values of the diagonal elements are determined by the dimension of the relevant $j, m$ subspaces, $\mathcal{H}_{j, m}$. Denoting the diagonal elements (populations) of $\rho_{n}^{\text {red }}$ by $p_{j, m}^{(n)}$, and using the

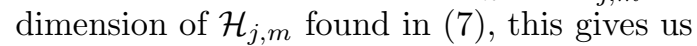

$$
p_{j, m}^{(n)}=\frac{\operatorname{dim} \mathcal{H}_{j, m}}{\operatorname{dim} \mathcal{H}}=2^{-n} D_{j}^{(1 / 2)} \quad \text { for } p=\frac{1}{2} .
$$

Finally, for this form of $\rho_{n}^{\text {red }}$ at $p=\frac{1}{2}$ to agree with the general $p$-dependence as found above, we find that $\rho_{n}^{\text {red }}$ must be diagonal in $j$ for all values of $p$ : Assume to the contrary that an off-diagonal element exists which is nonvanishing at some $p$-value. The required $p$-dependence implies that such a matrix element would also be nonvanishing at $p=\frac{1}{2}$ in contradiction with (11). Consequently such elements do not exist.

In conclusion, $\rho_{n}^{\text {red }}$ is completely diagonal, and the explicit form of the diagonal elements is

$$
p_{j, m}^{(n)}=\frac{2 j+1}{j_{0}+j+1}\left(\begin{array}{c}
2 j_{0} \\
j_{0}+j
\end{array}\right) p^{j_{0}+m}(1-p)^{j_{0}-m} .
$$

This surprisingly simple form of the full density matrix of an ensemble of identically distributed two-level systems immediately yields a host of interesting results.

As an important example we may use the result to illuminate the point that the states with less than full permutation symmetry are populated. First, the marginal $j$-distribution, $p_{j} \equiv \sum_{m} p_{j, m}^{(n)}$, is found to be

$$
p_{j}=D_{j}^{(1 / 2)} \frac{p^{2 j+1}-(1-p)^{2 j+1}}{2 p-1}(p(1-p))^{j_{0}-j} .
$$

For $p=\frac{1}{2}, p_{j}=(2 j+1) 2^{-n} D_{j}^{(1 / 2)}$ according to 111). For convenience we shall assume $p>\frac{1}{2}$ in the remainder of this section, and consequently it seems justifiable to approximate $(13)$ by dropping the term $(1-p)^{2 j+1}$ in the numerator, thus obtaining

$$
p_{j} \approx \frac{2 j+1}{j_{0}+j+1} \frac{p}{2 p-1}\left(\begin{array}{c}
2 j_{0} \\
j_{0}+j
\end{array}\right) p^{j_{0}+j}(1-p)^{j_{0}-j} .
$$

We observe that the rightmost three terms constitute a normalized binomial distribution with an expectation value for $j$ of $j_{c}=(2 p-1) j_{0}$ and a variance of $\sigma^{2}=$ $p(1-p) 2 j_{0}$. To justify the approximation made above in a more rigorous way, we may note that the omitted term corresponds to a similar binomial distribution centered at $-j_{c}$. The approximation is thus expected to be valid as long as $-j_{c}+\sigma<0$, or equivalently $p-\frac{1}{2}>1 / \sqrt{8 j_{0}}$.

The front-factor in the approximation (14) is slowly varying for $j$ not too close to 0 and it is close to unity at $j=j_{c}$, which suggests that we may describe the marginal $j$ distribution (13) by the binomial distribution alone. That this is indeed a good picture is illustrated in Fig. 1, where we also note that the limit of validity deducted above for the approximation (14) seems to be correct, as the only peak where the approximation deviates visibly from the exact value corresponds to $p=\frac{1}{2}+1 / \sqrt{8 j_{0}} \approx$ 0.535 chosen at the validity limit.

The variance in $j$ may be approximated by the variance of the binomial distribution, $\sigma^{2}$, which according to (4) is equal to $\Delta J_{z}^{2}$. Since $\sigma^{2}$ decreases with increasing $p$, we see that the $j$ states close to the maximal value of $j_{0}$ are only populated at very high polarizations, in agreement with Fig. 1. In particular the total population of the symmetric Dicke states $\left(j=j_{0}\right)$ is given by

$$
p_{j_{0}}=\frac{1}{2 p-1}\left(p^{n+1}-(1-p)^{n+1}\right),
$$

according to (12). To have $p_{j_{0}}>p_{j_{0}-1}$ we must have $1-p<1 / n$, corresponding to an expectation of finding less than one atom in the spin down state. Note, however, that already at $1-p<\log 2 / n$, we have half the total population in the symmetric Dicke states.

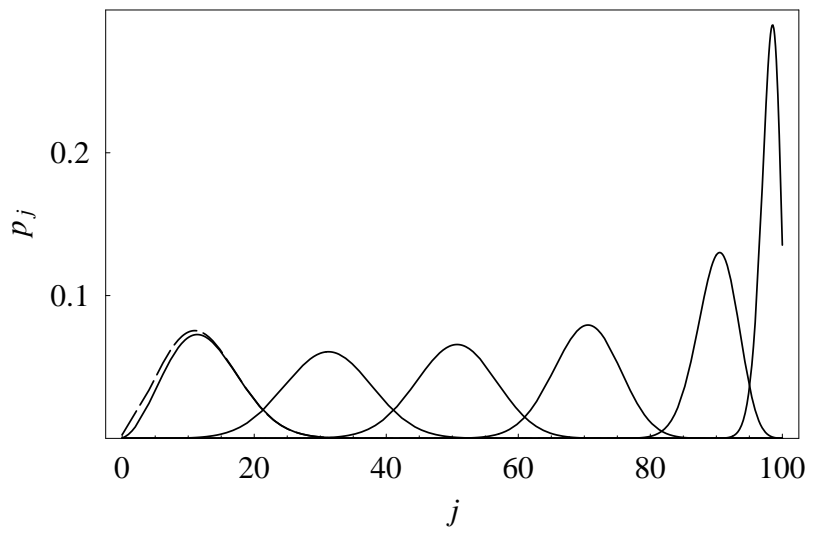

FIG. 1: The distribution of the collective spin of 200 spin $\frac{1}{2}$ particles at various polarizations. From left to right the peaks correspond to values of the polarization parameter $p$ in the single particle density matrix (1) of $0.535,0.65,0.75,0.85$, 0.95 and 0.99 respectively. The solid line is the exact value of $p_{j}$ according to (13), the dashed line corresponds to the approximation (14) which cannot not be discerned from the exact values at large $p$-values. Note that the $p=0.99$ line ending in mid-air is not a technical artifact, but illustrates a significant population of the symmetric Dicke states as given by $(15)$.

\section{THE EFFECT OF SPIN SQUEEZING ON A COLLECTION OF SPIN $\frac{1}{2}$ PARTICLES}

The variance of the spin components orthogonal to the mean spin of the state (8) obeys $\Delta J_{x} \Delta J_{y}=j_{0} / 2$, which exceeds the Heisenberg uncertainty limit, $\frac{1}{2}\left|\left\langle\left[J_{x}, J_{y}\right]\right\rangle\right|$, by a factor of $|2 p-1|^{-1}$, which approaches unity in the highly polarized cases $(p=0,1)$ as expected.

It is interesting to study the effect of spin squeezing operations on such states, since they might serve as models 
of imperfectly prepared initial states on which squeezing operations are performed. Note that obtaining a spin squeezed state indicates that the initially uncorrelated atoms described by (8) have become entangled [11].

Spin squeezing may be performed in a number of ways, and we will discuss the two unitary squeezing methods analyzed in Ref. 12] in the case of initially pure distributions. Both of these approaches make use of a Hamiltonian which is quadratic in collective spin variables, i.e., it involves interactions among the particles. The description of the evolution of the states would be immensely complicated in the product state representation of the many spins where, in fact, very entangled states are produced. In the collective spin representation, however, the action only has to be computed for each total spin component, which is a much easier task.

\section{A. One-axis twisting}

A one-axis twist about the $y$-axis, as mediated by the Hamiltonian $\chi J_{y}^{2}$, will squeeze the variances of the $J_{x}$ and $J_{y}$ spin components of an initially maximally polarized state as illustrated on Fig. 2 (the dotted ellipse): The variance of the spin along one direction in the $x y$-plane is increased, while the variance along the perpendicular direction is decreased to a value below the standard quantum limit $j_{0} / 2$.

As we have tried to illustrate by an example on the same figure, the same is qualitatively true in the case where the initial state is not a coherent spin state, but a "sufficiently polarized" mixed state.

Wave function components of definite $j$ show a reduced variance

$$
\Delta J_{\theta}^{2}=\frac{j}{2}\left(1-\frac{2 j-1}{4}\left[\sqrt{A_{j}^{2}+B_{j}^{2}}-A_{j}\right]\right)
$$

of the spin component $J_{\theta}=\cos \theta J_{y}-\sin \theta J_{x}$, where $\theta=$ $\frac{1}{2} \arctan \frac{B_{j}}{A_{j}}, A_{j}=1-\cos ^{2 j-2} \mu, B_{j}=4 \sin \frac{\mu}{2} \cos ^{2 j-2} \frac{\mu}{2}$ and $\mu=2 \chi t,[12]$. We note that both the optimum interaction time and the direction $\theta$ depend on $j$. For a distribution of $j$-values this will deteriorate the squeezing. Furthermore, the results in [12] assume an initial state with $m=j=j_{0}$, whereas we start out with a distribution of $m$-values.

Assuming that $p$ is so close to unity that we may use $j_{c}$ and $\sigma$ from section IIIB to estimate the center and width of the $j$-distribution, the relative variation of the optimal $\mu$ over the width of the $j$-distribution is approximately $\Delta \mu_{0} / \mu_{0} \approx-\sqrt{(1-p) / j_{0}}$. Consequently, for $j_{0} \gg 1$ it is possible to choose a value of $\mu$ that is nearly optimal for all populated $j$ subspaces. According to (12) and (13), we find that within each $j$-subspace, the fraction of the population in the uppermost $m$ level, $p_{j, j}^{(n)}$, is larger than $2-1 / p$.

Similarly the direction of the squeeze axis does not seem to vary significantly over the populated $j$ subspaces, as is also supported by Fig. 2 showing results for 15 atoms with $p=0.9$. Looking at the distributions within the different $j$-subspaces (dashed ellipses), one should note that the outermost ellipse corresponds not to the maximal $j$ but to $j=j_{0}-1$, which has the greatest population. $j_{0}$ correspond to the second-largest ellipse and we can see how the squeezing axis turns clockwise with increasing $j$. When we perform the sum over all $j$-values we obtain the result illustrated by the solid ellipse in Fig. 2. The state is significantly squeezed, but less than in the ideal case indicated by the dotted ellipse. As anticipated, the population of the states with non-maximal $m$ within each subspace does not appear to have serious adverse effect on the squeezing process.

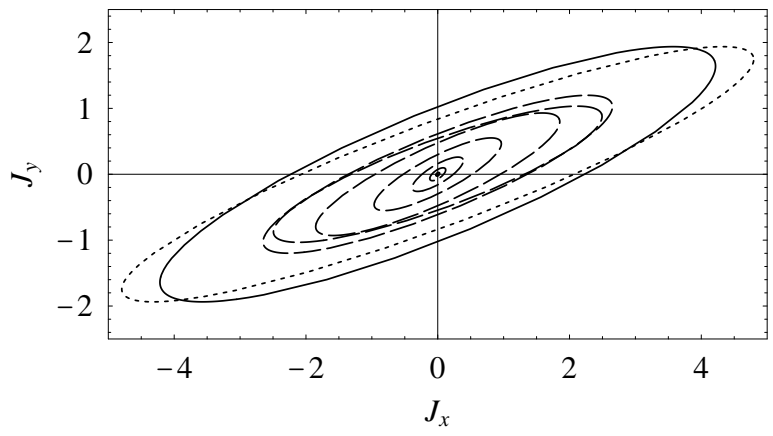

FIG. 2: The distribution of the spin components orthogonal to the mean spin after performing a one-axis twisting squeeze on a system of 15 spin $\frac{1}{2}$ particles with initial single particle density matrix given by (11) with $p=0.9$. The squared length of the minor and major axes of the ellipse drawn with a solid line equal the variance of the spin-component in these directions. Additionally the direction of the minor axis correspond to the direction of minimal variance. The dotted ellipse shows, for comparison, the result of applying an optimal squeezing to an initially pure system $(p=1)$, and the dashed ellipses illustrate the contributions from the various $j$ subspaces in the mixed case as discussed in the text.

\section{B. Two-axis countertwisting}

We analyze the time evolution under the two-axis countertwisting Hamiltonian, $H_{c t}=\chi / 2 i\left(J_{+}^{2}-J_{-}^{2}\right)$. One advantage of the two-axis countertwisting Hamiltonian is that the squeezing is always in the same direction. A disadvantage is that it cannot be solved analytically, and we will proceed according to the Holstein-Primakov approximation which is only valid for highly polarized samples and not too strong squeezing. Consequently we shall restrict the validity of our results to lowest order in $1-p$ and assume $j_{0}$ to be much larger than unity. Using results from [13] we find the variance of the squeezed component, $J_{y}$, of $\boldsymbol{J}$ after squeezing to be

$$
\Delta J_{y}{ }^{2}=\sum_{j, m} \rho_{j, m} \frac{1}{2}\left(j(j+1)-m^{2}\right) e^{-\lambda j},
$$


where $\lambda=4 \chi t$. In the fully polarized limit $(p=1)$ we have $\Delta J_{y}{ }^{2}=\exp \left(-\lambda j_{0}\right) j_{0} / 2$, and consequently $s_{0}=$ $\exp \left(\lambda j_{0} / 2\right)$ is the pure state squeezing factor.

It is a straightforward task to perform the sum over $m$ in (17). Doing this we obtain two terms inside the $j$-sum, one of which may safely be dropped under the assumptions already made $\left(p>\frac{1}{2}\right.$ and $\left.j_{0} \gg 1\right)$. Keeping the remaining term we find

$$
\begin{aligned}
\Delta J_{y}{ }^{2} \approx & e^{-\lambda j_{0}}\left(\frac{p}{p_{\lambda}}\right)^{2 j_{0}} \frac{p}{(2 p-1)^{2}} \\
& \sum_{j} \frac{j^{2}}{j+j_{0}}\left(\begin{array}{c}
2 j_{0} \\
j_{0}+j
\end{array}\right) p_{\lambda}{ }^{j_{0}+j}\left(1-p_{\lambda}\right)^{j_{0}-j},
\end{aligned}
$$

where we have used the asymptotic approximation, $j_{0} \gg$ 1 , extensively and introduced $p_{\lambda}$ as $p_{\lambda}=p /(1+(\exp (\lambda)-$ $1)(1-p))$. The three last terms of the second line are recognized as a normalized, rather narrow, binomial distribution. Approximating this distribution with a $\delta$ function centered at $j=\left(2 p_{\lambda}-1\right) j_{0}$ we find

$$
\Delta J_{y}{ }^{2} \approx e^{-\lambda j_{0}} \frac{j_{0}}{2}\left(\frac{p}{p_{\lambda}}\right)^{2 j_{0}+1}\left(\frac{2 p_{\lambda}-1}{2 p-1}\right)^{2} .
$$

To first order in $(1-p)$ this is equal to

$$
\Delta J_{y}^{2} \approx \frac{j_{0}}{2} e^{-\lambda j_{0}}\left(1+\left(e^{\lambda}-1\right)\left(2 j_{0}-3\right)(1-p)\right) .
$$

Based on this result we may determine the effect of the non-purity of the initial distribution on $\xi_{R}$ : the ratio between the frequency uncertainties obtainable by Ramsey spectroscopy on squeezed and unsqueezed samples, as introduced by Wineland et.al. [1]. According to this reference we have

$$
\xi_{R} \equiv \frac{\Delta \omega}{(\Delta \omega)_{0}}=\frac{\Delta J_{y} /\left|\left\langle J_{z}\right\rangle\right|}{\left(\Delta J_{y} /\left|\left\langle J_{z}\right\rangle\right|\right)_{0}},
$$

where the terms in the denominator refer to the unsqueezed sample. Since $\left(\Delta J_{y}^{2}\right)_{0}=j_{0} / 2$ is independent of $p$, and $\left\langle J_{z}\right\rangle=\left(\left\langle J_{z}\right\rangle\right)_{0}$ to first order in $\lambda$, we find that to lowest order in $(1-p)$ and $\lambda$ we have

$$
\xi_{R} \approx \frac{1}{s_{0}}\left(1+2(1-p) \log s_{0}\right),
$$

that is: to first order in $1-p$ we get a correction to the quality of the squeezed ensemble as compared to the case of performing the same squeezing operation on an initially perfectly polarized ensemble.

Note that we have only examined how imperfect initial state preparation affects the Ramsey spectroscopy; for an analysis of the significance of decoherence during the measurement see Refs. 14, 15.

\section{ATOMS WITH MORE THAN TWO LEVELS}

One of the virtues of the combinatorial analysis of collective spin distributions is that it may be generalized to the case where the atoms have $\operatorname{spin} s>\frac{1}{2}$.

\section{A. Collective spin states for $n$ spin $s$ particles}

Proceeding as in section III A we find that the dimension of the $\left|\begin{array}{l}j \\ m\end{array}\right\rangle$ subspace is

$$
D_{j}^{(s)}=\left(\begin{array}{c}
n \\
j_{0}+j
\end{array}\right)_{2 s}-\left(\begin{array}{c}
n \\
j_{0}+j+1
\end{array}\right)_{2 s}
$$

where the generalized binomial $\left(\begin{array}{c}n \\ m\end{array}\right)_{k}$ denotes the number of ways of placing $m$ indistinguishable balls in $n$ distinguishable urns so that no urn contains more than $k$ balls. Note that this definition agrees with that of the ordinary binomial for $k=1$.

The main problem with this approach is that the generalized binomials are not as analytically accessible as the binomials. Their numerical values may, however, easily be obtained through the recursion relation

$$
\left(\begin{array}{l}
n \\
m
\end{array}\right)_{k}=\sum_{i=0}^{k}\left(\begin{array}{l}
n-1 \\
m-i
\end{array}\right)_{k}
$$

and their analytical properties are to some extent accessible from the generating function:

$$
\begin{aligned}
\sum_{m}\left(\begin{array}{l}
n \\
m
\end{array}\right)_{k} t^{m} & =\left(1+t+\ldots+t^{k}\right)^{n} \\
& =\left(\frac{1-t^{k+1}}{1-t}\right)^{n} .
\end{aligned}
$$

\section{B. The density matrix for a tensor product of mixed states}

In order to represent the tensor product $\rho_{n}=\rho_{1}^{\otimes n}$ in the basis of eigenstates of the collective angular momentum, we have to develop this basis and to identify the matrix elements of $\rho_{n}$. It turns out to be advantageous to perform these two tasks in parallel by sequential coupling of angular momenta of $1,2, \ldots, k$ spin $s$ particles, and determination of the appropriate density matrices $\rho_{1}, \rho_{2}, \ldots, \rho_{k}$.

Let us introduce the collective spin of the first $k$ particles as

$$
\boldsymbol{J}^{(k)} \equiv \sum_{i=1}^{k} \boldsymbol{S}_{i}
$$

We denote by $\left\{\left|j_{1}, j_{2}, \ldots, j_{k}, m_{k}\right\rangle\right\}$ the simultaneous eigenstates of $\left(\boldsymbol{J}^{(1)}\right)^{2}, \ldots,\left(\boldsymbol{J}^{(k)}\right)^{2}$ and $J_{z}^{(k)}$ with eigenvalues $j_{1}\left(j_{1}+1\right), \ldots, j_{k}\left(j_{k}+1\right)$ and $m_{k}$ respectively. These states are obtained by sequential coupling of the atomic spins: by coupling the first, $j_{1}=s$, and second atom we obtain a total spin $j_{2}$, which is subsequently coupled with the third atom to the resulting spin $j_{3}$, etc.

The density matrix $\rho_{1}$ is our known input. We assume that $m_{s}$ labels the basis vectors in which $\rho_{1}$ is diagonal with eigenvalues $p_{m_{s}}$ (note that $m_{s}$ does not need to be 
associated with any real angular momentum or spin of the physical system, the spin representation is convenient to represent any $(2 s+1)$-level atomic system).

If we assume that the density matrix, $\rho_{k}$, of $k$ atoms is known in the basis of angular momentum coupled states, $\left\{\left|j_{1}, j_{2}, \ldots, j_{k}, m_{k}\right\rangle\right\}$, we can recursively construct the density matrix of $k+1$ atoms:

$$
\begin{aligned}
\rho_{k+1}= & \sum_{\alpha_{k}, \alpha_{k}^{\prime}, m_{k}, m_{s}}\left|\alpha_{k}^{\prime}, m_{k}\right\rangle\left\langle\alpha_{k}, m_{k}|\otimes| \stackrel{s}{m_{s}}\right\rangle\left\langle\stackrel{s}{m_{s}}\right| \\
& \left\langle\alpha_{k}^{\prime}, m_{k}\left|\rho_{k}\right| \alpha_{k}, m_{k}\right\rangle p_{m_{s}},
\end{aligned}
$$

where we have utilized that $\rho_{k}$ is diagonal in $m_{k}$ as argued in section IIIB, and we have introduced $\alpha_{k}$ as a shorthand for the intermediate angular momenta $j_{1}, j_{2}, \ldots, j_{k}$. It is now just a matter of angular momentum coupling to obtain from (26) the density matrix $\rho_{k+1}$ in the basis of total angular momentum states of the $k+1$ particles, specifying $j_{k+1}, m_{k+1}$ and all the intermediate angular momenta $\alpha_{k}$.

The bookkeeping seems to be a demanding problem, but as in Eq. (9) we are only interested in the reduced density matrix retaining the properties of the total spin of the $k+1$ particles. Hence we trace over $\alpha_{k}$, i.e., we add incoherently the contributions from subspaces with different intermediate $j_{k^{\prime}}$ : Let $p_{j_{k}, m_{k}}^{(k)}$ denote the diagonal elements of the reduced density matrix $\rho_{k}^{\mathrm{red}}$, that is, the probability that $k$ atoms have a total angular momentum of $j_{k}$ and projection $m_{k}$. We can then write the matrix elements of the reduced density matrix for $k+1$ atoms:

$$
\begin{aligned}
& \left\langle\begin{array}{c}
j_{k+1}^{\prime} \\
m_{k+1}^{\prime}
\end{array}\left|\rho_{k+1}^{\text {red }}\right| \begin{array}{c}
j_{k+1} \\
m_{k+1}
\end{array}\right\rangle=
\end{aligned}
$$

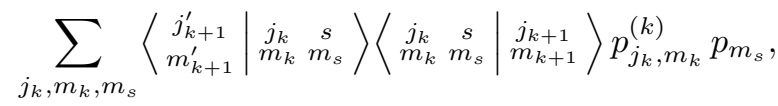

where the brackets, $\left\langle\begin{array}{cc|c}j_{k} & s \\ m_{k} & m_{s} & j_{k+1} \\ m_{k+1}\end{array}\right\rangle$, denote ClebschGordan coefficients. An interesting consequence of the relation (27) is that for $k$ less than $n$ it is sufficient to determine recursively the diagonal elements of the reduced density matrix, $\rho_{k}^{\text {red }}: p_{j_{k}, m_{k}}^{(k)}=$ $\sum_{j_{k-1}, m_{k-1}, m_{s}}\left|\left\langle\begin{array}{cc}j_{k-1} & s \\ m_{k-1} & m_{s}\end{array} \mid \begin{array}{l}j_{k} \\ m_{k}\end{array}\right\rangle\right|^{2} p_{j_{k-1}, m_{k-1}}^{(k-1)} p_{m_{s}}$. In the last step we obtain $\rho_{n}^{\text {red }}$ which also has coherences between different collective spin states. According to the selection rules of angular momentum coupling this implies that the reduced density matrix must be diagonal in $m_{n}$ and band diagonal in $j_{n}$ with values ranging over $\left|\Delta j_{n}\right| \leq 2 s$.

As an example, consider two spin 1 particles which are both in the pure state $\left|\begin{array}{l}1 \\ 0\end{array}\right\rangle$. In the collective spin basis the combined state of these particles is

$$
\left|\begin{array}{l}
1 \\
0
\end{array}\right\rangle \otimes\left|\begin{array}{l}
1 \\
0
\end{array}\right\rangle=\sqrt{\frac{2}{3}}\left|\begin{array}{l}
2 \\
0
\end{array}\right\rangle-\sqrt{\frac{1}{3}}\left|{ }_{0}^{0}\right\rangle,
$$

which clearly yields a non-diagonal reduced density matrix.

Note that according to (12) we actually have the stronger selection rule $\Delta j=0<2 s$ for $s=\frac{1}{2}$.

In closing, we present a simple case of a mixed state of a system of uncorrelated atoms with more than two levels, which might serve as an approximation of e.g., an optically pumped system. We assume the single atom density matrix, $\rho_{1}$, to be diagonal, and of the form

$$
p_{m_{s}}=\frac{1}{Z} e^{-\beta m_{s}},
$$

where $Z$ is a normalization constant depending on the parameter $\beta$. By induction through (27), or by the argument that we used to deduce the $p$ dependence in section IIIB, we find that with this form of $\rho_{1}$, the parameter dependence of the reduced density matrix, $\rho_{n}^{\text {red }}$, must be $Z^{-n} \exp (-\beta m)$. By the same arguments as in section IIIB we conclude that the reduced density matrix is diagonal and

$$
p_{j, m}^{(n)}=D_{j}^{(s)} Z^{-n} e^{-\beta m},
$$

where $D_{j}^{(s)}$ is given by (22).

\section{SUMMARY}

In conclusion, we have reiterated the convenience of collective spin operators for analyzing the behavior of a system of atoms.

We have established the explicit form of the reduced density matrices of ensembles of two and multi-level atoms with a given single particle distribution, and we have used these explicit distributions to predict the effect of non-perfect sample preparation on the effectiveness of spin squeezing by one-axis twisting and two-axis countertwisting.

\section{Acknowledgments}

This work was supported by the Danish Research Foundation - Danmarks Grundforskningsfond and by the Information Society Technologies Programme IST2000-30064, REQC.
[1] D. J. Wineland, J. J. Bollinger, W. M. Itano, and D. J. Heinzen, Phys. Rev. A 50, 67 (1994).

[2] M. A. Nielsen and I. L. Chuang, Quantum Computaion and Quantum Information (Cambridge University Press, 2000).

[3] A. Kuzmich, K. Mølmer, and E. S. Polzik, Phys. Rev. 
Lett. 79, 4782 (1997).

[4] J. Hald, J. L. Sørensen, C. Schori, and E. S. Polzik, Phys. Rev. Lett. 83, 1319 (1999).

[5] L.-M. Duan, A. Sørensen, J. I. Cirac, and P. Zoller, Phys. Rev. Lett. 85, 3991 (2000).

[6] A. Sørensen, L.-M. Duan, J. I. Cirac, and P. Zoller, Nature 409, 63 (2001).

[7] I. Bouchoule and K. Mølmer, To appear in Phys. Rev. A 2002, quant-ph/0105144.

[8] A. Kuzmich, L. Mandel, and N. P. Bigelow, Phys. Rev. Lett. 85, 1594 (2000).

[9] B. Julsgaard, A. Kozhekin, and E. S. Polzik, Nature 413, 400 (2001).
[10] F. T. Arecchi, E. Courtens, R. Gilmore, and H. Thomas, Phys. Rev. A 6, 2211 (1972).

[11] A. Sørensen and K. Mølmer, Phys. Rev. Lett. 86, 4431 (2001).

[12] M. Kitagawa and M. Ueda, Phys. Rev. A 47, 5138 (1993).

[13] L. Mandel and E. Wolf, Optical Coherence and Quantum Optics (Cambridge University Press, 1995), chap. 21.3.

[14] S. F. Huelga, C. Macchiavello, T. Pellizzari, A. K. Ekert, M. B. Plenio, and J. I. Cirac, Phys. Rev. Lett. 79, 3865 (1997).

[15] D. Ulam-Orgikh and M. Kitagawa, Phys. Rev. A 64, 052106 (2001). 